\title{
Lidil
}

Revue de linguistique et de didactique des langues

Altérité et formation des enseignants

\section{Négociation perceptive et altérité en classe de langues}

Anne Feunteun et Diana-Lee Simon

\section{OpenEdition}

\section{Journals}

Édition électronique

URL : http://journals.openedition.org/lidil/3126

DOI : 10.4000/lidil.3126

ISSN : $1960-6052$

\section{Éditeur}

UGA Éditions/Université Grenoble Alpes

\section{Édition imprimée}

Date de publication : 1 mai 2009

ISBN : 978-2-84310-139-7

ISSN : $1146-6480$

\section{Référence électronique}

Anne Feunteun et Diana-Lee Simon, « Négociation perceptive et altérité en classe de langues », Lidil [En ligne], 39 | 2009, mis en ligne le 26 juin 2018, consulté le 01 mai 2019. URL : http:// journals.openedition.org/lidil/3126 ; DOI : 10.4000/lidil.3126 


\title{
Négociation perceptive et altérité en classe de langues
}

\author{
Anne FEUNTEUN * et Diana-Lee SIMON**
}

\begin{abstract}
RÉSUMÉ
Cet article s'appuie principalement sur une recherche en didactique des langues (Feunteun, 2007) qui donne à voir qu'un travail guidé, mené par les maitres avec les élèves de cycle 2, sur l'expression partagée et négociée de leurs perceptions, au contact de langues variées peut favoriser, sur la durée, une décentration perceptive linguistique et culturelle. On observe que ce choix de démarche réflexive partagée interroge fortement les enseignants sur leur propre capacité à écouter, accueillir, interpréter et médiatiser la parole de leurs élèves, en séance de langue(s) et à tout moment dans la classe, ouvrant ainsi des pistes à développer dans le cadre de la formation initiale et continue des enseignants.
\end{abstract}

\section{ABSTRACT}

This article, inspired by recent research in the field of language didactics (Feunteun, 2007), aims at showing that guided work by teachers with young pupils aged 5 to 7 in "cycle 2" encouraging mutual expression and negotiation of their perceptions in activities involving several languages, can contribute in the long term to linguistic and cultural distancing in relation to those perceptions. The study highlights the way in which the choice of such a reflexive, sharing approach strongly questions teachers with regard to their own capacity to listen, to be welcoming and accepting, to interpret and mediate the utterances of their pupils, both within the lessons devoted to language learning and indeed at all times in class, thus opening new avenues to be pursued in pre-and in-service teacher education.

En prenant appui sur une recherche récente en didactique des langues (Feunteun, 2007), cet article propose comme point de départ de mettre en évidence la manière dont des enfants de cinq à sept ans perçoivent

* IUFM Orléans-Tours, Dynadiv.

** Université Stendhal-Grenoble 3, Lidilem. 
« l'étrangeté » des langues qu'ils rencontrent dans le cadre des activités de classe. Il s'intéresse au rôle de l'enseignant dans la co-construction des échanges destinés à accueillir les perceptions exprimées, et à la nature « interculturelle » de la négociation perceptive qui s'ensuit. À ce titre, il postule l'existence d'un espace de négociation perceptive, dorénavant ENP, qui serait le lieu privilégié où s'effectue pour des enfants de cet âge un travail sur l'altérité et donc d'ouverture à l'autre. L'enseignant se trouve ainsi, en situation pédagogique, mis au défi d'écouter, d'accueillir, d'interpréter et de médiatiser les perceptions, ce travail se révélant parfois délicat et difficile. L'analyse des représentations des enfants, mais aussi les témoignages des enseignants impliqués dessinent en creux des questions importantes pour la formation des enseignants.

La réflexion que nous proposons s'inscrit dans la perspective actuelle du développement du plurilinguisme en contexte scolaire, étayée par de nombreuses recherches et publications ${ }^{1}$. Ce foisonnement de travaux s'explique par une certaine tension entre la volonté d'œuvrer pour de la mise en œuvre d'une politique linguistique du plurilinguisme et du pluriculturalisme dans l'esprit du Cadre européen commun de référence pour les langues (désormais CECRL) et le fait que l'école française en particulier, mais aussi celle d'autres pays européens, n'est pas un lieu historiquement ou institutionnellement ouvert au plurilinguisme et au pluriculturalisme ${ }^{2}$. Ce phénomène renvoie au constat que bon nombre d'élèves côtoient plusieurs langues au quotidien, mais que celles-ci restent bien souvent ignorées et inexploitées dans la classe (Dinvaut, 2008, p. 331). Par ailleurs, les familles de certains enfants censurent leurs propres langues au profit de la langue de l'école (le français, langue de solarisation, langue d'intégration). Sur le plan institutionnel et pédagogique, les freins au développement d'une éducation au plurilinguisme sont de plusieurs ordres : les textes officiels incitent les enseignants à se concentrer sur la langue de l'école, « langue nationale » et langue véhiculaire des apprentissages scolaires; les approches méthodologiques et curriculaires restent souvent très cloisonnées (le français du « socle commun des connaissances » et une

1. Candelier (2003), Candelier et al. (2008), Castellotti, Coste et Duverger (2008), Castellotti (2001 et 2008), Moore (2006), Perregaux et al. (2003), entre autres.

2. Coste, Moore et Zarate (1997 et 1998, p. 31-32) soulignent ce point dans les réflexions fondatrices de la conception des compétences plurilingues et pluriculturelles avancée dans le CECRL. 
« langue vivante étrangère »); le parcours de formation des enseignants ne les prépare pas fondamentalement à accueillir les cultures en présence dans leur classe.

La recherche-action menée par Anne Feunteun (2007) sur une période de trois ans, fut l'occasion d'impulser sur le terrain scolaire en cycle 2 , une démarche pédagogique intégrée fondée sur la diversité linguistique et culturelle. Celle-ci se construit à partir d'activités articulant langue de l'école (le français), langue étrangère ${ }^{3}$ (l'anglais bien souvent) et éveil aux langues et cultures (musiques du monde, comptines et chansons, ateliers réflexifs). L'hypothèse que nous avançons est que l'expression partagée et négociée des perceptions des enfants lors de ces activités, va favoriser une réorganisation potentielle des représentations de chacun, contribuant ainsi à l'entrouverture et à un début de décentration.

Nous présenterons brièvement le contexte de la recherche-action, les acteurs impliqués dans l'expérimentation (apprenants et enseignants), la démarche de recherche et le mode de traitement des données. Suivra une discussion théorique pour éclairer le choix terminologique d'espace de négociation perceptive, (ENP) central dans cette recherche. On observera alors certaines traces du fonctionnement de cet ENP à travers une analyse des représentations des enfants, tout en mettant en évidence les témoignages de leurs enseignants confrontés aux perceptions particulières exprimées par leurs jeunes élèves. Notre conclusion questionnera de nouvelles orientations pour la formation des enseignants.

\section{Présentation de la recherche didactique (Feunteun, 2007)}

\section{Contexte et méthodologie de recherche}

La recherche-action s'est déroulée sur une période de trois ans entre 2003 et 2006 dans les classes de cycle 2 de deux écoles du Loir et Cher et a porté sur 128 élèves et 5 enseignants. L'analyse qualitative des observables s'est appuyée sur des enregistrements réguliers de séances de classes entières où les tâches et les échanges étaient orientés de façon

3. Nous utilisons le terme « langue étrangère » en cohérence avec les Instructions officielles pour les langues à l'école, tout en soulignant que pour les enfants, l'anglais est une langue plus ou moins « étrangère » dans la mesure où tous sont exposés aux contacts médiatiques divers avec cette langue. 
à favoriser l'émergence de représentations sur les apprentissages et l'expression partagée de leurs expériences perceptives provoquées par les langues et les cultures en contact. Des dessins faits par des groupes d'élèves ou par des individus et commentés, soit en interaction entre pairs, soit en entretiens avec la chercheure, ont fourni des données intéressantes sur le travail de négociation concernant les perceptions, comme nous le verrons ci-dessous.

\section{Les acteurs : apprenants et enseignants}

\section{Les apprenants}

L'expérimentation a débuté par des entretiens ${ }^{4}$ menés avec les enfants de 5 à 7 ans, de la grande section de maternelle au CE1. Ceuxci ont révélé que contrairement à l'image d'enfants " monolingues » entretenue par les parents et par les maîtres en début de recherche, ces enfants côtoyaient d'autres langues hors de l'espace scolaire, notamment les langues d'origine de leurs parents ou grands-parents - en l'occurrence l'anglais, l'anglais australien, l'arabe algérien, l'arabe berbère, l'arabe marocain, l'arabe syrien, plusieurs langues de Guinée, l'espagnol, le chinois, mandarin, le japonais, le laotien, le polonais, le portugais, le turc, le tchétchène, le géorgien ou l'arménien, pour l'ensemble interrogé. Cette impressionnante diversité de langues et cultures constitutives du répertoire de chacun (le déjà-là), a pu être exploitée dans le cadre de la démarche pédagogique retenue. La question de recherche s'est alors orientée vers la possibilité (et le bien-fondé) de faire évoluer les représentations des enfants vers un accueil empathique de toute langue culture.

4. Feunteun fait par ailleurs le constat que contrairement à ce qu'on l'on peut communément lire depuis 1989, les très jeunes enfants de cinq à sept ans ne sont ni des «éponges », ni des apprenants « efficaces » car décrétés «malléables ». Elle découvre que les enfants ont souvent anticipé de manière très personnelle, selon leur héritage socioculturel propre, sur la façon dont ils vont et devraient apprendre la langue de l'école mais aussi la langue étrangère : l'anglais. Ces premières attentes des enfants s'articulent sur des représentations de la langue et de la culture de l'autre déjà présentes. 


\section{Les enseignants}

Sur les cinq enseignantes impliquées dans la recherche, trois ont accepté d'adopter une approche plurielle ${ }^{5}$ des langues et des cultures (Candelier, 2008), alors que les deux autres ont refusé de s'investir dans la démarche, soit par crainte de ne pas «être à la hauteur en anglais » ou parce qu'il y avait déjà «fort à faire à leur apprendre le français ». Aucune des enseignantes expérimentatrices n'avait de cursus de linguiste, mais elles avaient en commun un souvenir douloureux d'apprentissage scolaire de l'anglais. L'innovation mise en place les a amenées à questionner leur propre culture didactique en tant que praticiens réflexifs (Vasseur et Grandcolas, 1999 ; Perrenoud, 2003), contribuant ainsi à la dynamisation du contexte, et à une amorce d'innovation.

\section{Origines du concept espace de négociation perceptive $(\text { ENP })^{6}$}

Le concept de l'ENP est complexe, car il renvoie à une spécificité de l'activité sociale d'apprentissage chez les enfants très jeunes, dont les perceptions exprimées en classe sont fortement ancrées dans le réel de leur vie sensorielle ${ }^{7}$ (gout, ouie, vue, toucher). Il s'inscrit aussi dans la conception de la classe comme une communauté de pratiques, riche de savoirs et de vécus multiples (linguistiques, culturels, expérientiels) telle que Mercer $(1996,2000)$ et Lave et Wenger (1991) la définissent. Dans cette perspective, l'apprenant est un interactant social qui apprend dans la rencontre, l'écoute et la confrontation d'opinions ou d'expressions perceptives issues de l'expérience des pairs et de l'enseignant. Selon les propos de Mercer (2000, p. 6), «grâce au langage, le potentiel mental de chacun va pouvoir s'articuler en une intelligence communicative collective qui va permettre à tous de mieux comprendre le monde et de confronter les meilleures façons de l'appréhender ». Ces

5. En particulier l'appropriation de l'anglais en articulation avec l'apprentissage du français.

6. Ce terme est issu de la recherche de Feunteun (2007, p. 38).

7. À titre d'exemple, Lucie, en grande section, confie en entretien : «j'aime bien star/ça veut dire étoile/mais ça brille plus quand on l'dit en anglais », alors qu'Aurore en CE1 entendant de l'espagnol en « Éveil aux Langues » s'écrie : «les mots i'sentent bon comme les gâteaux de ma voisine ». 
échanges, qu'il appelle "exploratory talk ${ }^{8}(2000$, p. 98$)$, mènent à la création du «savoir». Enfin, notre définition tient compte de processus « intermental» ou « interthinking» (Mercer, 2000, p. 141). En d'autres mots, «pour rendre possible l'enseignement pour le maitre et l'appropriation pour l'apprenant, ils doivent s'engager mutuellement dans l'échange au sein d'une activité conjointe afin de créer un espace de communication. Dans cette zone de mise en synergie de pensée, qui ne cesse d'être redéfinie par et dans le dialogue, apprenant et enseignant co-construisent leur cheminement par et dans la négociation ».

\section{Fonctionnement de l'ENP : traces de la négociation perceptive}

Les représentations, qu'elles soient « prêt-à-penser » ou « prêt-à-dire », sont au cœur du processus de l'altérité dans la mesure où elles constituent les matériaux langagiers "mobilisés » par les acteurs dans les échanges entre pairs « pour catégoriser une expérience nouvelle, ramener l'inconnu dans des cadres interprétatifs préétablis », (Castellotti, 2001, p. 24), bref, appréhender et accueillir l'étrangeté.

\section{Rechercher du semblable pour accéder à l'inconnu}

Encouragés vers l'autonomie stratégique, les enfants, confrontés à l'inconnu, recherchent du semblable ou de l'associable comme dans l'exemple ci-dessous. Au moment des fêtes de fin d'année, la classe de $\mathrm{CP}$ a découvert que, pour certains enfants, Noël était un jour ordinaire sur leur calendrier. $\mathrm{Ba}$, une élève chinoise, accompagnée de sa mère, a été invitée à présenter le nouvel an chinois et les élèves ont observé avec intérêt «son » calendrier. Ils ont ensuite découvert, en les mimant et mémorisant à l'oral, deux comptines (une en chinois et une en anglais) qui désignent les traits du visage. Spontanément, ils ont cherché à associer et à comparer les sons et les signes ${ }^{9}$ nouvellement découverts, aux sons et aux mots formés de lettres en anglais en s'appuyant sur la langue de l'école pour discuter de leurs dessins.

8. Un échange verbal exploratoire. Les pairs critiquent de manière constructive les idées de l'autre, faisant émerger de manière tangible le raisonnement.

9. Ils n'ont pas eu de difficulté à identifier la graphie du chinois en la comparant à ce qu'ils avaient vu sur le calendrier de $\mathrm{Ba}$. 


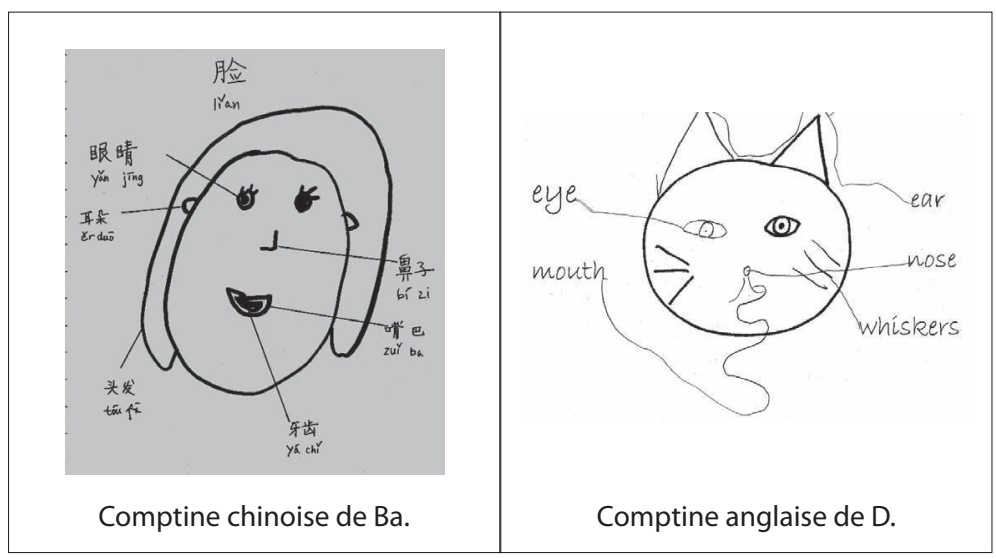

Fig. - Dessins d'enfants. Découverte des comptines des enfants de la classe à l'issue des ateliers cuisine.

Voici un extrait des commentaires libres des enfants, lorsque ces derniers entament un travail de négociation perceptive en comparant les dimensions graphie-phonie de langues éloignées pour un premier accueil sensoriel et réfléchi de la nouveauté :

Arthur 1 - [yan jing] ça veut dire two eyes/

Noémie 1 - ben oui i'a deux mots/ pareils

Arthur 2 - normal y a deux yeux

Noémie 2 - oui mais même / y a pas l'même dessin

Arthur 3 - ben c'est [one- ya] et [yan- two] et en chinois les sons ça s'dessine pas pareil :

Noémie 3 - faudrait d'mander à $\mathrm{Ba}$ / elle elle sait comme nous en anglais et en français mais elle est née chinois

Les enfants placent leurs savoirs multiples au centre de leurs négociations partagées (" et puis avec tout ce qu'on sait déjà/on s'débrouille/ » dira un enfant de fin de cycle 2). De leurs premiers essais d'associations plurilingues nait un questionnement sans blocage, car sans autocensure normée (Calvet, 1999). La langue de l'école est alors le médium commun d'étonnement, d'échanges pour rassurer, tandis que la langue étrangère enseignée joue un rôle de passerelle entre les langues familières de la classe et les autres langues du monde. Des langues lointaines par leurs écritures et leurs paysages sonores peuvent être ainsi approchées, apprivoisées, et rapprochées. 


\section{Quand l'étrangeté rebute et fait obstacle}

Certaines fois, la perception de l'étrangeté s'exprime dans un mouvement de rejet à travers une mise-en-relation d'éléments du langage avec une perception visuelle ou sonore. C'est ainsi que l'enseignant peut entendre un élève dire : - «en anglais y a trop de lettres c'est comme eux /i' sont trop gros » (expression d'antipathie), ou encore : «y avait des mots grossiers» (une perception sonore mal accueillie et péjorative). Ce qui est remarquable dans ces exemples, c'est le passage métonymique entre la langue et ceux qui la parlent, qui est le plus direct, fournissant une occasion pédagogique intéressante à relever pour un travail sur ces stéréotypes. D'autres élèves soulignent la différence au niveau de la qualité sonore perçue : «ça fait un peu hachoté / ça lolotte » ou encore : "quand on entend c'est gluant ou pas comme nous ». La xénité (Weinrich, 1989), ressentie comme dérangeante, peut même faire obstacle à la mémorisation : « quand ça dérange/on l'garde pas dans la tête ». Dans ces cas, l'enseignant est témoin de l'expression de préjugés négatifs exprimant la différence, l'invitant à participer à un travail d'expression partagée de ces représentations afin d'œuvrer dans le sens de l'ouverture à l'autre, de l'acceptation de la différence dans le microcosme de la classe.

\section{Comparaisons perceptives interlinguales et altérité}

Des échanges entre enfants lors d'une séance d'éveil aux langues et aux cultures offrent un exemple précieux de la manière insolite dont cette co-construction négociée peut offrir une amorce de décentration :

\footnotetext{
Pierre 4 - en français ça cogne pas/ ça monte pas trop non plus/

Tony 1 - ben c'est pour ça que l'français/ c'est pas une langue étrangère/

Cyrille 2 - pour les anglais/ peut-être que le français/ i' l'entendent bizarre aussi/

Pierre 5 - oui mais ça fait pas des vagues/ alors ça cogne pas dans leurs oreilles/
}

$\mathrm{Au}$ niveau de leurs représentations sociales, les enfants recourent à des «microthéories prêtes à l'emploi », pour reprendre l'expression de Py (2004, p. 8), afin d'exprimer leur appartenance à un même groupe quand ils sont confrontés à "l'étranger». De toute évidence Pierre fait une comparaison indirecte entre les sonorités «familières » de la langue de l'école, et "l'étrangeté » sonore de la langue étrangère, l'anglais. Son oreille fine détecte des différences au niveau de 
l'accentuation et de la prosodie, mais aussi au niveau de la qualité des sonorités qu'il trouve dures en anglais, car elles « cognent » dans (ses) oreilles. Tony confirme que le français, n'est pas pour eux, une langue étrangère. Toutefois, Cyrille fait preuve d'ouverture et de perspicacité, car elle arrive à se mettre à la place de l'autre, en imaginant que les Anglais puissent de leur position ou point de vue, percevoir le français comme étant «bizarre » et elle invite donc ses camarades à remettre en cause leur position, une invitation à se décentrer. Comme Pierre reste campé sur ses positions, on voit bien la potentialité pour l'enseignant de poursuivre le travail amorcé dans l'ENP. Dans cette zone de mise en synergie de pensée, par et dans la négociation, il y aurait lieu de poursuivre les échanges dans une démarche pédagogique plus « construite et réfléchie » pour l'enseignant(e), mais y est-il/elle préparé(e) ou prêt(e)?

\section{Accepter la différence de langues... et de gouts}

De son côté, un petit garçon de CE1 exprime, lors d'une séance en classe qui a donné lieu à des échanges avec les pairs de cultures familiales plurielles, qu'il peut tout à fait accepter intellectuellement que les autres parlent et vivent différemment, mais il souhaite réaffirmer, en aparté, sa centration sur son mode alimentaire. Il exprime par là sa perception de proximité et de distance culturelle :

\begin{tabular}{|c|c|}
\hline noît & $\begin{array}{l}\text { t dans toutes les langues/ il y a des choses } \\
\text { sont étrangères/ qui sont étranges }\end{array}$ \\
\hline Tom & $\begin{array}{l}\text { aît pas/ comme l'arabe/ mais après c'e } \\
\text { uand on sait/ }\end{array}$ \\
\hline & $\begin{array}{l}\text { (en aparté, très sérieusement à son voisin) pour la lang } \\
\text { mais pas pour le manger. }\end{array}$ \\
\hline
\end{tabular}

À sept ans, un travail sur l'accueil empathique de l'autre est possible et souhaitable. Accepter de gouter à la cuisine de l'autre est un grand pas, l'adopter n'est pas une obligation. Reconnaitre, au cycle 2, que pour les autres ce soit bon, même si mon palais n'apprécie pas, n'est-ce pas là l'équilibre à rechercher entre décentration et centration ? Comme le souligne Morin (1999, p. 145), «l'importance du choix d'attitude montre que ce qui est en jeu, c'est bien le regard que l'on porte sur l'altérité et sur le monde ». Le fait de percevoir des variations langagières et culturelles ne signifie pas pour autant les faire siennes, de manière radicale. Le type de démarche que les enfants de cycle 2 ont pu vivre les invite à relativiser plus qu'à intégrer, à négocier pour ne pas exclure ou 
pour ne pas subir ce que l'on ne comprend pas, ce qui dérange. Gérer les échanges dans cet ENP n'est pas forcément évident pour l'enseignant.

\section{Se percevoir comme étant " un autre » pour l'autre}

Dans le travail transversal d'éveil aux langues et cultures, les groupes classes parviennent, à leur rythme, à appréhender la notion que l'autre est un sujet. Mais que l'on soit soi-même un autre pour l'autre, voilà qui est compliqué. Les enfants font toutefois preuve d'une perspicacité qui étonne l'adulte. Ayant écouté sa camarade tchétchène relater son expérience plurilingue un élève conclut :

Hamed 93 - en fait/ on est étranger tous les deux/ quand on joue avec un enfant qu'a un accent ou qui parle pas français lui aussi i'doit trouver qu'on fait des trucs pas comme lui/ on est étranger tous les deux.

Ou encore l'enfant qui réalise en commentant son dessin (Feunteun 2007, p. 504) :

Ce qui est drôle, c'est que nous aussi on peut paraitre étranger pour quelqu'un d'autre, alors là je vais rajouter des arbres sur ma tête comme des antennes, ça veut dire que toi tu te trouves normal, mais quand l'autre il va t'entendre, c'est toi qui deviens étranger.

Ces commentaires invitent enseignante et élèves au cœur même du processus interculturel, de la négociation de l'entre-cultures. On peut y entendre l'écho de la notion d'altérité telle que Laplantine (1999, p. 32-33) l'énonce et qui renvoie à l'idée que l'étrangeté est en nous. Les dires des enfants qui émergent dans l'ENP sont un point de départ fécond pour un travail dans la communauté de la classe sur l'accueil et l'acceptation de la différence, si urgent dans notre société actuelle, mais ce travail ne s'improvise pas et nécessiterait d'être conforté par la formation des enseignants.

\section{Témoignages d'enseignants et questions de formation}

Les échanges des apprenants analysés ci-dessus ont permis d'esquisser en creux quelques traits relatifs au rôle potentiel du maitre dans le processus de négociation perceptive à l'œuvre pour de très jeunes enfants engagés dans une approche plurielle des langues. Comme le dit Glissant (1996), pour que l'enseignant puisse aider l'apprenant à se reconnaitre et à s'accepter comme «multiple » possible, encore faut-il 
que lui-même soit prêt pour cette démarche individuelle et collective, dans sa classe. Se pose alors la question épineuse de la formation des enseignants à la relation à l'altérité. Comment les préparer de manière adéquate pour mener à bien ce travail qui s'avère complexe et délicat ?

Le fonctionnement de l'ENP a été observé principalement par rapport aux échanges entre pairs, dans la perspective de l'évolution de leurs répertoires. Comme toute activité initiée dans l'ENP de chaque enfant peut être associée à ce que Lave et Wenger (1991, p. 50) nomment « un réseau de relations en évolution et transformation constantes ${ }^{10}, l^{\prime}$ 'enseignant est au cœur du tissage de ces relations. L'ENP fonctionne donc également au niveau plus large de l'espace classe et des échanges auxquels participe l'enseignant. C'est ainsi que nous avons vu émerger à travers les dires des enfants, des moments potentiellement féconds ${ }^{11}$ pour le travail de co-construction des savoirs, suggérant un espace pour le discours de l'enseignant dans le processus de négociation perceptive initié parfois spontanément par les enfants, et qui fonctionne comme une adresse à leur enseignant pour un travail sur les perceptions, sur les résistances, sur l'altérité. Ce travail est à construire, bien évidemment aussi en formation initiale et continue, et nous ne sous-estimons pas la complexité de la tâche.

Les enseignantes « expérimentatrices » dans cette recherche-action ont vécu une «formation sur le tas », ou une formation «in situ », qui, bien que limitée à la praxis - y associant néanmoins une dimension réflexive ${ }^{12}$ dans le temps - a tout de même permis d'enclencher une évolution de leurs pratiques et de leurs représentations, et pour certaines, une prise de conscience des exigences et nouveautés d'une

10. Milroy (1980) définit le réseau social d'un locuteur comme la somme de ses relations individuelles avec d'autres personnes et les relations de ces autres personnes entre elles. Différents critères permettent d'apprécier la structure du réseau. Le maillage, plus ou moins dense, des relations dépend de l'importance et de la qualité des solidarités latérales. « Ce maillage [...] et sa densité influencent les comportements linguistiques des membres du réseau. » Voir aussi Milroy et Milroy (1985).

11. Ces moments potentiellement féconds pour la négociation perceptive rappellent le concept de «séquences potentiellement acquisitionnelles » qui ouvrent des espaces propices à l'apprentissage.

12. L'enseignant s'interroge, en amont de ses pratiques mais aussi au fil de ses vécus de classe, sur ce qu'il pourrait mettre en place comme «situation didactique » (Castellotti et De Carlo, 1995) pour favoriser et dynamiser l'appropriation partagée de savoirs et de savoir-faire. 
telle démarche pédagogique somme toute novatrice. Elsa, l'enseignante de CE1, par exemple, montre qu'une telle démarche induit des changements de rôles et de places dans la classe qui peuvent être déstablisants, car l'enseignant n'est plus seul détenteur du savoir, des savoirs :

[...] en classe entière : en éveil aux langues/ en arts plastiques/en tout tout tout/ on a toujours travaillé comme ça/ça veut dire aussi que le maitre n'est plus celui qui sait tout : qui peut tout prévoir : c'est comme dans les entretiens/ c'est une écoute active très intense/ il faut penser vite et laisser faire/--/ en fait on les aide à penser ensemble/ mais on pense /on vit l'échange avec eux/ c'est très intense/ on apprend en quelque sorte à vivre et à apprendre ensemble/ en classe/ par ce que l'on écoute et ce que l'on dit [...].

L'activité d'apprentissage dépend de l'écoute et des dires de chacun, pris en compte dans le processus de négociation perceptive et ne va pas sans remise en cause par rapport aux pratiques « antérieures » ou « habituelles », comme l'exprime Coralie :

ça t'interroge drôl'ment/en fait le système produit une hiérarchisation des langues et des cultures/ et en fait : on le cautionne :: sans y penser vraiment/ on fait/ on parle pas avec les enfants de ce qu'ils ressentent quand ils apprennent/ quand ils écoutent/ c'est pas évident [...].

Analysant son propre cheminement lors d'un entretien à l'issue des trois ans de pratique réflexive, Claudie devient consciente de sa propre évolution biographique et didactique :

[...] je ne pourrais plus enseigner comme avant/ au début je croyais que c'était juste faire des ponts entre le français et l'anglais// les faire réfléchir à comment ils apprennent/ comment ils perçoivent/ ça a permis à des enfants de parler d'eux-mêmes/ ça m'a obligé à me taire tout en étant très vigilante/on a tous appris à s'écouter et à réfléchir à c'qu'on dit d'habitude/ et moi à c'que j'fais [...].

L'étude suggère qu'il y a une expansion possible à la fois des répertoires biographiques et didactiques des maitres et des biographies plurielles des enfants. Cette dynamique prend son essor dans les représentations co-construites que l'on se fait des autres et de l'ailleurs, initiant, dans la classe, mode de pensée et attitudes favorables à l'accueil interpersonnel, culturel et langagier. L'ENP se révèle être ainsi un lieu privilégié pour engager un travail de fond sur l'altérité linguistique et culturelle, pourvu bien évidemment, que les enseignants soient à même de s'y atteler, ce qui implique une formation en adéquation avec la mise en œuvre d'approches plurielles. 


\section{Conclusion}

Cette réflexion centrée sur le travail de la négociation perceptive à l'œuvre lors d'activités de compréhension avec de très jeunes élèves confrontés à la diversité linguistique et culturelle, a permis de mettre en lumière toute l'importance de la dimension relationnelle à l'altérité au cœur du processus d'apprentissage des langues. Il serait tout à fait intéressant de développer à l'avenir des tâches de production afin d'élargir le champ d'investigation. Par ailleurs, il serait intéressant d'interroger le concept d'ENP en termes de « démarche pédagogique ». Si les expérimentations analysées invitent fortement à envisager une « démarche pédagogique de négociation perceptive » dans les classes, une réflexion complémentaire serait à mener permettant de dégager des pistes, des caractéristiques exploitables à des fins de formation. Sans vouloir forcément en faire un "outil pédagogique » au sens où l'on entend habituellement ce terme, la vraie question nous semble celle de savoir comment enrichir le profil personnel et professionnel de chaque enseignant, en formation initiale et continue, pour qu'il prenne le risque au quotidien, dans sa classe, de guider ses élèves dans leur négociation perceptive de l'altérité.

Nous tenons là un fil précieux qui permet une articulation forte avec les attentes exprimées dans le CECRL, où l'on peut lire qu' ' il s'agit surtout d'aider les apprenants à construire leur identité langagière et culturelle en y intégrant une expérience diversifiée de l'altérité, et à développer leurs capacités d'apprenants à travers cette même expérience diversifiée de l'altérité » (Conseil de l'Europe, 2001, p. 105). Cette démarche ouvre une voie prometteuse permettant de lier les finalités scolaires à une éducation langagière plus ouverte à la pluralité des langues et des cultures (Castellotti, Coste et Duverger, 2008). L'ENP, décliné à l'échelle de la classe, devient alors un espace à cultiver pour que chaque acteur, enfant et adulte, puisse y co-construire des pans de son identité sociale, linguistique et culturelle.

\section{Remerciements}

Nous remercions chaleureusement Louise Dabène et nos relecteurs anonymes pour leurs remarques utiles. 


\section{RÉFÉRENCES BIBLIOGRAPHIQUES}

Calvet L.-J. (1993) : Sociolinguistique, Paris, PUF.

Calvet L.-J. (1999) : Pour une écologie des langues du monde, SaintAmand-Montrond, Plon.

CANDElier M. (2003) : L'éveil aux langues à l'école primaire. Evlang: bilan d'une innovation européenne, Bruxelles, De Boeck.

CAndelier M. et al., (2008) : Conscience du plurilinguisme - Pratiques, Représentations et Interventions, Rennes, PU, Des Sociétés.

CAstellotti V. (2001) : D'une langue à d'autres : pratiques et représentations, Rouen, Collection Dyalang, UMR CNRS 6065, PUR.

Castellotti V., Coste D. et Duverger J. (dir.) (2008) : Propositions pour une éducation au plurilinguisme en contexte scolaire, Paris, ADEB / DYNAVIV.

Castellotti V. et De Carlo M. (1995) : La formation des enseignants de langue, Didactique des langues étrangères, Paris, CLE international.

Coste D., Moore D., et Zarate G. (1997) : La compétence plurilingue et pluriculturelle. Langues vivantes. Vers un cadre européen commun de référence pour l'enseignement et l'apprentissage des langues vivantes, Études préparatoires, Strasbourg, Éditions du Conseil de l'Europe.

CONSEIL DE L'EuROPE (2001) : Cadre européen commun de référence pour les langues, apprendre, enseigner, évaluer, Paris, Didier.

Dinvaut A. (2008) : La didactique plurilingue des langues : obstacles et propositions, Thèse de Doctorat de sciences du langage, Université Lumière Lyon 2.

Feunteun A. (2007) : S'approprier des langues à l'école, une co-construction perceptive chez des enfants de cinq à sept ans, Thèse de Doctorat, Université de Tours.

Glissant E. (1996) : Introduction à la poétique du divers, Paris, Gallimard.

Laplantine F. (1999): Je, nous et les autres. Être humain au-delà des appartenances, Paris, Le Pommier-Fayard.

Lave J. et Wenger E. (1991) : Situated Learning. Legitimate peripheral participation, Cambridge, University of Cambridge Press.

MERCER N. (1996) : « The quality of talk in children's collaborative activity in the classroom », Learning and Instruction, 6 (4), p. 359-377.

Mercer N. (2000) : "Language for teaching a language», dans C. N. Candlin et N. Mercer, English Language Teaching in its Social Context, New York, Macquarie University \& The Open University.

Milroy L. (1980): Language and Social Networks, Oxford, Basil Blackwell.

Milroy J. et Milroy L. (1985) : Authority in Language. Investigating Language Prescription and Standardisation, New York, Routeledge \& Kegan Paul. 
Moore D. (2006) : Plurilinguismes et école, Paris, Didier.

Morin E. (1999) : Relier les connaissances ; le défi du XXI e siècle, Seuil.

Perdereau-Bilski M.-P. (2005) : « Des savoirs cachés aux savoirs acquis : quand les premiers étayent les seconds dans la construction de compétences croisées », dans Glottopol, Revue de sociolinguistique en ligne, $\mathrm{n}^{\circ}$ 6. Disponible sur <http://www.univ-rouen.fr/dyalang/glottopol> (consulté le 25 août 2006).

Perregaux C., de Goumens C., Jeannot D. et De Pietro J.-F. (2003) : É $d u$ cation et ouverture aux langues à l'école, vol. 1 et 2, Neuchâtel, CIIP.

Perrenoud P. (2001/2003) : Développer la pratique réflexive dans le métier d'enseignant, Professionnalisation et raison pédagogique, Pédagogies, Recherche, ESF.

Py B. (2004): «Pour une approche linguistique des représentations sociales », Langages, 154, p. 6-19.

Vasseur M. T. et Grandcolas B. (1999): Conscience d'enseignant, conscience, d'apprenant, réflexions interactives pour la formation, Publication Socrates-Lingua Action A, Paris, Université René Descartes-Paris 5.

Weinrich H. (1989) : «Les langues les différences », Le Français dans le monde, 228. 
